\title{
EFFECT OF NONLINEAR RADIATIVE HEAT AND MASS TRANSFER ON MHD FLOW OVER A STRETCHING SURFACE WITH VARIABLE CONDUCTIVITY AND VISCOSITY
}

\author{
Akindele M. Okedoye ${ }^{1}$, Sulyman O. Salawu ${ }^{2}$ \\ ${ }^{1}$ Department of Mathematics and Computer Science, Federal University of Petroleum \\ Resources, Effurun, Nigeria \\ ${ }^{2}$ Department of Mathematics, Landmark University, Omu-aran, Nigeria \\ e-mail: kunlesalawu2@gmail.com \\ *Corresponding author
}

\begin{abstract}
A theoretical investigation on the flow of nonlinear magnetohydrodynamic (MHD), laminar, viscous, incompressible boundary layer fluid with thermal radiative heat transfer and variable properties past a stretching plate was carried out. The liquid is taken to be gray, absorbing, emitting but with non-scattering medium. The main nonlinear equations governing the flow are reduced to ordinary differential equations by using appropriate similarity variables and quantities. The obtained nonlinear equations are computationally solved by applying shooting techniques coupled with Nachtsheim-Swigert method for asymptotic satisfaction of boundary conditions by fourth order Runge-Kutta scheme. The computational results for momentum and heat distribution are obtained for various values of the emerging parameters. The results for the coefficient of skin friction and dimensionless heat gradient are likewise obtained for different physical parameters values. From the study, it was observed that the parameters which enhanced the heat source terms decreased the fluid viscosity and caused increase in the flow rate. Also, parameter that reduced heat source terms encouraged viscosity which resulted in retardation of the fluid velocity.
\end{abstract}

Keywords: Power-law velocity; Stretching surface; MHD flow; Nonlinear radiation; variable properties

Nomenclature

(x) - Variable applied magnetic induction

$\theta-$ Dimensionless temperature

$\mathrm{T}$-Temperature of the fluid

$\mathrm{q}_{\mathrm{r}}$ - Component of radiative flux

$\mathrm{m}$-Velocity exponent parameter

$c_{p}-$ Heat capacity

$\mathrm{p}$-Pressure of the fluid

$\mathrm{k}_{*}-$ Thermal conductivity

$\rho-$ Density of the fluid

$\Psi$ - Stream function

v -Kinematic viscosity

$\mathrm{T}_{\mathrm{w}}$ - Heated plate temperature 
$\mathrm{B}_{0}-$ Constant applied magnetic induction

$\mathrm{T}_{\infty}$-Fluid ambient temperature

$\mathrm{u}, \mathrm{v}-$ Velocity component of fluid in $\mathrm{x}$ and $\mathrm{y}$ direction

\section{Introduction}

It is an established contention that several industrial and engineering processes involving heat and mass transport such as glass fibre, metal extrusion, rubber manufacturing, and many more takes place in the presence of simultaneous effects of thermal and species buoyancy forces. Different studies on the fluid flow through an inclined, horizontal and vertical surface in the existence of magnetic field have been examined. In Seth et al. (2011) and Salawu and Fatunmibi (2017) the analysis of dissipative heat transfer of hydromagnetic fluid flow past an inclined plate was investigated. Salawu and Okedoye (2018) investigated gravity driven flow of reactive hydromagnetic fluid through a vertical channel in the presence of magnetic field. Hassan et al. (2018), Kareem et al. (2020) studied hydromagnetic reactive fluid flow through horizontal porous plates with radiation and internal heat generation.

Fluids flow past a vertical Couette boundary layer plates with heat radiation is gaining considerable attraction due to its wide spectrum usefulness in industrial systems. For example, it useful in the rocket engine, combustion chamber, geothermal reservoirs, thermal insulation, and so on. Various leading past studies concerning convection flow through vertical plates in the presence radiation has been established in the research work of (Hayat et al. (2013); Salawu et al. (2018); Devi and Gururaj (2012)). The heat transfer in over a stretching plate is important in several devices and industrial applications. In manufacturing process of rubber and plastic sheet where it is frequently important to blow gaseous through the unsolidified material, this circumstance emerge in the glass blowing, expulsion processes, and spinning of fibers also include the flow as a result of stretching plate, Hayat et al. (2013). Devi and Gururaj (2012) reported on heat transport characteristics of two dimensional nonlinear hydromagnetic incompressible fluids with variable viscosity and electrically conductivity. The liquid is taken to be gray, absorbing, emitting but not scattering medium. In Patowary (2012), the influence of radiation on the flow of boundary layer in the existence of magnetic field with thermal conductivity and variable viscosity due to a stretching surface in a permeable medium was investigated. To show the heat flux by radiation in the heat equation, Rosseland approximation was utilized. The energy and species transport through a vertical surface under the joined effect of the diffusion thermo and thermo diffusion in the existence of magnetic field was carried out by Hazarika and Gopal (2012). A computational solution of a convective transient fluid flow with thermal radiation over a moving plate of a Sisko binary fluid was analyzed by Okedoye (2015). The problem formulated was solved numerically; the outcomes of the analysis demonstrated that the flow is affected considerably by the injection/suction, heat source, and chemical reaction at the plate surface. Also, the impact of soret and dufour on a Sisko fluid is significant.

The understanding of the thermophysics properties of some parameters associated with the fluid flows with temperature dependent variable properties have instant influences on the micro fluidics, ink-jet printing, polymer production, earth mantle geological flows, colloidal flow suspensions, turbulent flow shear, fluid gems, and many more, Salawu and Oke (2018). In respect of this, scientist has being showing high interest on the intrigue behavior of fluids with variable properties and subsequently studies has been done on it using the analytical and computational approaches which are available in many articles including (Salawu (2018); Abdou (2010); Salem (2007); Chen (2006)). A few great reports on the flow of fluid through a stretching surface are presented by (Modather et al. (2007); Yurusoy (2006); Pantokratoras, A (2006)). Considering the above studies, the authors ignored the heat dependent thermal conductivity and viscosity in the momentum variation power-law. The variable physical properties may vary meaningfully with 
changes in temperature, when taken into variable properties into consideration. The present study aims to investigate heat dependent variable properties with variable surface velocity over a stretching plate. The flow is propelled by the influence of buoyancy forces in the existence of thermal radiation in a stretching plate.

\section{Formulation of the problem}

Forced convection flow of nonlinear radiation along a stretching horizontal plate kept at the same wall temperature $T_{w}$. The stretching surface is with velocity power law of $u_{w}=u_{0} x^{m}$ (where $\mathrm{u}_{0}$ and $\mathrm{m}$ are constants) through a fluid with variable viscosity is considered. The electrically conducting fluid is taken to be incompressible, viscous, absorbing, emitting, gray and nonscattering medium with temperature $\mathrm{T}_{\infty}$. A magnetic field is assumed perpendicular to the flow in a horizontal stretching surface as depicted in (Figure 1) according to Devi and David (2012). Cartesian coordinate system is chosen. The main flow is along $\mathrm{x}$-axis direction in a stretching sheet with velocity components $u$ and $v$ in these directions (Salawu and Dada (2018); Salem (2007)).

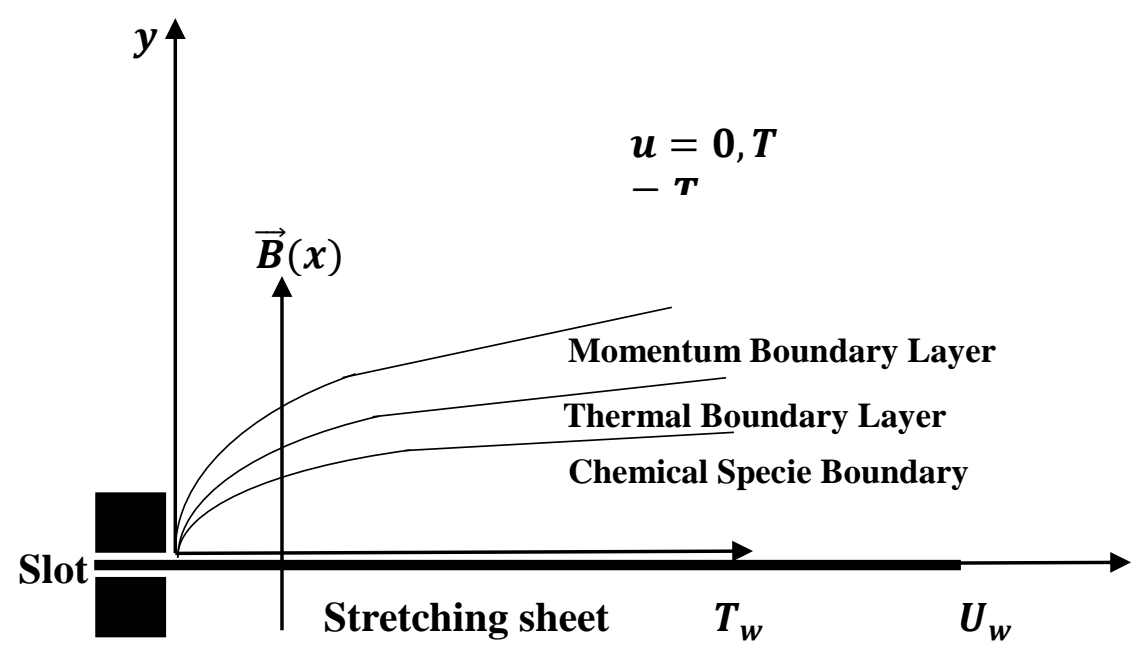

Fig. 1. Schematic diagram of the problem

The flow runs continuously along the $\mathrm{x}$-axis with $\mathrm{y}$-axis normal to it. The assumptions below are considered:

- The flow is laminar, steady in two-dimensional.

- Excluding the viscosity of the fluid, the fluid thermophysical properties are taken be unchanged.

- The induced magnetic and Reynolds number are considered negligible and small respectively.

- $\quad$ The Joule's heating and heat viscous is taken to be negligible. 
- $\quad$ The heat flux radiation is considered negligible in the $\mathrm{x}$-direction compared to the $\mathrm{y}$ direction.

The continuity, momentum, and energy conservation equations under the above assumptions are presented as:

$$
\begin{gathered}
\frac{\partial u}{\partial x}+\frac{\partial u}{\partial y}=0 \\
\rho\left(u \frac{\partial u}{\partial x}+v \frac{\partial u}{\partial y}\right)=\frac{\partial}{\partial z}\left(\mu \frac{\partial u}{\partial y}\right)+g \beta \tau\left(T-T_{\infty}\right)+g \beta c\left(C-C_{\infty}\right)-\sigma B_{0}^{2} u \\
\rho c_{p}\left(u \frac{\partial T}{\partial x}+v \frac{\partial T}{\partial y}\right)=\frac{\partial}{\partial y}\left(k_{*} \frac{\partial T}{\partial y}\right)+\frac{\partial q_{r}}{\partial y} \\
\rho\left(u \frac{\partial C}{\partial x}+v \frac{\partial C}{\partial y}\right)=\frac{\partial^{2} C}{\partial y^{2}}+A\left(C-C_{\infty}\right)
\end{gathered}
$$

The fluid viscosity is taken as

$$
\frac{1}{\mu}=d_{2}\left(T-T_{r}\right)
$$

Where

$$
d_{2}=\frac{d_{1}}{\mu_{\infty}}, T_{r}=T_{\infty}-\frac{1}{d_{1}}, \text { and } B(x)=B_{0} x^{\frac{m-1}{2}}
$$

Here, $d_{1}, d_{2}$ and $T_{r}$ are constants, and their values depend on the fluid thermal property and the reference state.

The associated boundary conditions are

$$
\begin{aligned}
& u=u_{w}=u_{0} x^{m}, v=0, T=T_{w} \text { at } y=0\left(u_{0}>0\right) \\
& u=0, \\
& T=T_{\infty} \text { as } y \rightarrow \infty
\end{aligned}
$$

where $u_{w}$ is the velocity of the stretching surface, the components velocity are respectively the quantities $u$ and $v$ in the direction of $x$ and $y, u_{0}$ is a constant, $B_{0}$ is the magnetic field and all the other quantities have their usual meanings.

The heat flux radiative term is defined by using the Rosseland diffusion approximation, Salawu and Dada (2018);

$$
q_{r}=-\frac{16 \sigma^{*} T^{3}}{3 \alpha^{*}} \frac{\partial T}{\partial y}
$$

where $\sigma^{*}$ is the Boltzmann-Stefan constant, $\alpha^{*}$ is the coefficient mean Rosseland absorption. Assume the temperature difference within the flow are sufficiently small such that $T^{4}$ may be expressed as a linear function of temperature, using Taylor series to expand $T^{4}$ about the free stream $T_{\infty}$ and neglecting higher order terms, this gives the approximation $T^{4} \cong 4 T_{\infty}^{3} T-3 T_{\infty}^{4}$. Using the stream function $\psi(\mathrm{x}, \mathrm{y})$, the continuity equation is satisfied such that 


$$
u=\frac{\partial \psi}{\partial y} \text { and } v=-\frac{\partial \psi}{\partial x}
$$

Applying the suitable similarity transformation, Salem (2007)

$$
\begin{gathered}
\eta(x, y)=y \sqrt{\frac{m+1}{2}} \sqrt{\frac{u_{0} x^{m-1}}{v}} \\
\psi(x, y)=y \sqrt{\frac{2}{m+1}} \sqrt{v u_{0} x^{m+1}} f(\eta) \\
\theta(\eta)=\frac{T-T_{\infty}}{T_{w}-T_{\infty}}, \theta_{w}=\frac{T_{w}}{T_{\infty}}
\end{gathered}
$$

where $\theta_{\mathrm{w}}$ is surface temperature parameter, equation (7) is redefined using appropriate variables. Now

$$
\begin{aligned}
q_{r} & =-\frac{16 \sigma^{*} T^{3}}{3 \alpha^{*}} \frac{\partial T}{\partial y} \\
& =-\frac{16 \sigma^{*} T^{3}}{3 \alpha^{*}}\left(T_{w}-T_{\infty}\right) \sqrt{\frac{m+1}{2}} \sqrt{\frac{u_{0} x^{m-1}}{v}}\left(\left(\frac{T_{w}}{T_{\infty}}-1\right) \theta+1\right)^{3}\left(\frac{T_{w}}{T_{\infty}}-1\right) \frac{d \theta}{d \eta} \\
& =-\frac{16 \sigma^{*} T^{4}}{3 \alpha^{*}} \sqrt{\frac{m+1}{2}} \sqrt{\frac{u_{0} x^{m-1}}{v}}\left(\left(\frac{T_{w}}{T_{\infty}}-1\right) \theta+1\right)^{3}\left(\frac{T_{w}}{T_{\infty}}-1\right)^{2} \frac{d \theta}{d \eta}
\end{aligned}
$$

Since

$$
\begin{gathered}
\theta_{w}=\frac{T_{w}}{T_{\infty}}, \text { let } \theta_{w}=\bar{\theta}_{w}+1 \\
q_{r}=-\frac{16 \sigma^{*} T^{4}}{3 \alpha^{*}} \sqrt{\frac{m+1}{2} \frac{u_{0} x^{m-1}}{v}}\left(\theta \bar{\theta}_{w}+1\right)^{3} \bar{\theta}_{w}^{2} \frac{d \theta}{d \eta}
\end{gathered}
$$

Thus,

$$
\begin{gathered}
\frac{\partial q_{r}}{\partial y}=\frac{d q_{r}}{d \eta} \frac{d \eta}{d y}=-\frac{16 \sigma^{*} T^{4}}{3 \alpha^{*}} \frac{m+1}{2} \frac{u_{0} x^{m-1}}{v} \bar{\theta}_{w}\left(3 \bar{\theta}_{w}\left(\theta \bar{\theta}_{w}+1\right)^{2} \theta^{\prime 2}+\left(\theta \bar{\theta}_{w}+1\right)^{3} \theta^{\prime \prime}\right) \\
\frac{\partial q_{r}}{\partial y}=-\frac{16 \sigma^{*} T^{4}}{3 \alpha^{*}} \frac{m+1}{2} \frac{u_{0} x^{m-1}}{v} \bar{\theta}_{w}\left(3 \bar{\theta}_{w}\left(\theta \bar{\theta}_{w}+1\right)^{2} \theta^{\prime 2}+\left(\theta \bar{\theta}_{w}+1\right)^{3} \theta^{\prime \prime}\right)
\end{gathered}
$$

Hence, equation (8) is the transformed dimensionless radiation. It is also obtained that

$$
\begin{aligned}
\frac{1}{\mu} & =d_{2}\left(T-T_{r}\right)=\frac{1}{\mu_{\infty}}\left(\left(T-T_{\infty}\right) \gamma+1\right)=\frac{1}{\mu_{\infty}}\left(\left(T-T_{\infty}\right) \gamma \theta+1\right) \\
& =\frac{\left(T_{w}-T_{\infty}\right) \gamma}{\mu_{\infty}}\left(\theta+\frac{1}{\left(T_{w}-T_{\infty}\right) \gamma}\right)=b\left(T_{w}-T_{\infty}\right)\left(\theta+\theta_{c}\right)
\end{aligned}
$$




$$
\frac{1}{\mu}=b\left(T_{w}-T_{\infty}\right)\left(\theta+\theta_{c}\right)
$$

Further simplification of equation (9) is expressed as

$$
\begin{gathered}
\frac{1}{k_{*}}=\frac{1}{k_{\infty}}\left(1+k\left(T-T_{\infty}\right)\right)=\frac{1}{k_{\infty}}\left(1+k\left(T_{w}-T_{\infty}\right) \theta\right) \\
=\frac{k}{k_{\infty}}\left(T_{w}-T_{\infty}\right)\left(\theta+\frac{1}{k\left(T_{w}-T_{\infty}\right)}\right)=\alpha\left(\theta+\theta_{r}\right) \\
\frac{1}{k_{*}}=\alpha\left(\theta+\theta_{r}\right)
\end{gathered}
$$

Therefore, the heat dependent variable viscosity is redefined in equation (10)

Viscosity factor $b=\frac{\gamma}{\mu_{\infty}}\left\{\begin{array}{l}>0 \text { for liquids } \\ <0 \text { for gases }\end{array}\right.$,

$$
\theta_{c}=\frac{1}{\left(T_{w}-T_{\infty}\right) \gamma}, \theta_{r}=\frac{1}{k\left(T_{w}-T_{\infty}\right)}, \alpha=\frac{k}{k_{\infty}}\left(T_{w}-T_{\infty}\right)
$$

Then

$$
\begin{aligned}
& v=-\frac{\partial \psi}{\partial x}=-\sqrt{\frac{m+1}{2}} \sqrt{u_{0} x^{m-1}}\left(f+\frac{m-1}{m+1} \eta f^{\prime}\right) \\
& \text { and } \\
& u=\frac{\partial \psi}{\partial y}=u_{0} x^{m} f^{\prime}
\end{aligned}
$$

Thus continuity equation (1) implies $m \equiv 1$, therefore, the non-dimensional variables appropriate for the problem under consideration are:

$$
\eta=y \sqrt{\frac{u_{0}}{v}}, v=-\sqrt{u_{0} v} f, u=u_{0} x f^{\prime}
$$

Using equation (11) on the equations (2) - (4) and boundary condition (6) along with equations (8) to (10). Then we have

$$
\begin{gathered}
\frac{\theta_{c}}{\theta+\theta_{c}} f^{\prime \prime \prime}+f f f^{\prime \prime}-f^{\prime 2}-\frac{\theta_{c}}{\left(\theta+\theta_{c}\right)^{2}} \theta^{\prime} f^{\prime \prime}+\operatorname{Grt} \theta+G r c \phi-M f^{\prime}=0 \\
\frac{\theta_{r}}{\theta+\theta_{r}} \theta^{\prime \prime}+\operatorname{Prf} \theta^{\prime}-\frac{\theta_{r}}{\left(\theta+\theta_{r}\right)^{2}} \theta^{\prime 2}+\frac{4 \bar{\theta}_{w}}{3 R}\left(3 \bar{\theta}_{w} \theta^{\prime 2}+\left(\bar{\theta}_{w} \theta+1\right) \theta^{\prime \prime}\right)\left(\bar{\theta}_{w} \theta+1\right)^{2}=0 \\
\phi^{\prime \prime}+\operatorname{Scf} \phi^{\prime}+\beta \operatorname{Sc} \phi=0 \\
f(0)=0, f^{\prime}(0)=1, \phi(0)=1, \theta(0)=1: y=0 \\
\theta(\eta) \rightarrow 0, \phi(\eta) \rightarrow 0, f^{\prime}(\eta) \rightarrow 0: \eta \rightarrow \infty
\end{gathered}
$$


The equations (12) to (15) are the dimensionless flow equations, where $\theta_{c}=\frac{1}{\left(\mathrm{~T}_{\mathrm{w}}-\mathrm{T}_{\infty}\right) \gamma}$ is the viscosity measuring parameter. $\theta_{\mathrm{r}}=\frac{1}{\mathrm{k}\left(\mathrm{T}_{\mathrm{W}}-\mathrm{T}_{\infty}\right)}$ is the thermal conductivity term, $\mathrm{R}=\frac{\mathrm{k} \alpha^{*}}{4 \sigma^{*} \mathrm{~T}^{4}}$ is the heat transfer rate, $M=\frac{\sigma B_{0}^{2}}{\rho u_{0}}$ is the magnetic term, $\frac{\mu c_{p}}{k}$ is the Prandtl number, Grt $=\frac{g \beta_{\tau}\left(T_{W}-T_{\infty}\right)}{\rho x u_{0}^{2}}$ is the thermal buoyancy, $\operatorname{Grc}=\frac{g \beta_{\tau}\left(C_{w}-C_{\infty}\right)}{\rho \mathrm{xu}_{0}^{2}}$ is the mass buoyancy, $\mathrm{Sc}=\frac{\mu}{\mathrm{Dm}}$ is the Schmidt number, $\beta=\frac{\mathrm{A}}{\mathrm{vu}_{0}}$ is the reaction parameter.

\section{Numerical solution of the problem}

Computational solution to the dimensionless equations (12)-(14) along with the boundary conditions (15) are obtained by coupled trapezoid method with Runge-Kutta seventh-eighth of continuous order scheme and Richardson extrapolation improvement (Rani et al. (2013); Sharidan et al. (2006); Kareem et al. (2018), Salawu and Ogunseye (2020)). A shooting method is used first to change the derivatives to differential equations of first order. The non-magnetic solution is taken as the primary guess; the Euler iterative techniques is used continuously until it convergence within the given precision. The following are prescribed parameters in the study $\beta$ , Grt, Grc, M, R, $\theta_{w}, \phi, \theta_{c}, \theta_{r}, \mathrm{Sc}$, and Pr. The initial guesses with the equation was solved by Thomas' algorithm. The computation was carried out using MAPLE 18 software.

\subsection{Special Cases}

(i) In nonexistence of radiation term, the numerical value is compared to that of Devi and David (2012) as illustrated through Tables 1 and 2. From the tables, it is noticed that the results are in good agreement with that of Devi and David (2012).

(ii) When magnetic field is absent, the results obtained in the study takes the form Okedoye et al. (2017).

(iii) In the absence of variable conductivity and magnetic field, the study is similar to that of Okedoye (2015) in the absence of radiation effect with constant thermal conductivity.

\subsection{Validity of Results}

We compare this result with the result of Devi and David (2012) with our numerical result for $\mathrm{M}=2$ and $\mathrm{M}=4$. It is observed from Table 1 and 2 that the numerical values of velocity $f^{\prime}(\eta)$ and $\theta(\eta)$ is in good agreement as shown in tables.

\begin{tabular}{|c|c|c|c|c|c|c|}
\hline $\mathrm{f}^{\prime}(\eta)$ & \multicolumn{3}{|c|}{ M= 2.0 } & \multicolumn{3}{c|}{ M = 4.0 } \\
\hline$\eta$ & $\begin{array}{c}\text { Devi \& } \\
\text { David }\end{array}$ & $\begin{array}{c}\text { Current } \\
\text { Work }\end{array}$ & $\begin{array}{c}\text { Differenc } \\
\text { e }\end{array}$ & $\begin{array}{c}\text { Devi \& } \\
\text { David }\end{array}$ & $\begin{array}{c}\text { Current } \\
\text { work }\end{array}$ & Difference \\
\hline & & 1.0000000 & 0.0000000 & 1.0000000 & 1.0000000 & 0.0000000 \\
0.0 & 1.00000000 & 0 & & 0 & 0 & 0 \\
\hline & & 0.3233977 & 0.0000000 & 0.2332171 & 0.2338171 & 0.0000000 \\
0.4 & 0.32339773 & 3 & & 5 & 6 & 1 \\
\hline & & 0.1073692 & 0.0000000 & 0.0561298 & 0.0565298 & 0.0000000 \\
0.8 & 0.10736922 & 2 & & 3 & 3 & 0 \\
\hline & & 0.0366827 & 0.0000009 & 0.0139944 & 0.0137947 & 0.0000003 \\
1.2 & 0.03668281 & 2 & & 8 & 8 & 0 \\
\hline
\end{tabular}




\begin{tabular}{|c|c|c|c|c|c|c|}
\hline 1.6 & 0.01282269 & $\begin{array}{c}0.0128226 \\
9\end{array}$ & 0.0000000 & $\begin{array}{c}0.0036779 \\
1\end{array}$ & $\begin{array}{c}0.0030780 \\
1\end{array}$ & $\begin{array}{c}0.0000001 \\
0\end{array}$ \\
\hline 2.0 & 0.00466227 & $\begin{array}{c}0.0046622 \\
8\end{array}$ & 0.0000010 & $\begin{array}{c}0.0009622 \\
0\end{array}$ & $\begin{array}{c}0.0006621 \\
0\end{array}$ & $\begin{array}{c}0.0000001 \\
0\end{array}$ \\
\hline 2.4 & 0.00175143 & $\begin{array}{c}0.0017515 \\
4\end{array}$ & 0.0000011 & $\begin{array}{c}0.0002922 \\
0\end{array}$ & $\begin{array}{c}0.0006925 \\
0\end{array}$ & $\begin{array}{c}0.0000003 \\
0\end{array}$ \\
\hline 2.8 & 0.00060943 & $\begin{array}{c}0.0006096 \\
3\end{array}$ & 0.0000020 & $\begin{array}{c}0.0000815 \\
2\end{array}$ & $\begin{array}{c}0.0007820 \\
2\end{array}$ & $\begin{array}{c}0.0000005 \\
0\end{array}$ \\
\hline 3.2 & 0.00023224 & $\begin{array}{c}0.0002322 \\
4\end{array}$ & 0.0000000 & $\begin{array}{c}0.0000379 \\
4\end{array}$ & $\begin{array}{c}0.0002381 \\
4\end{array}$ & $\begin{array}{c}0.0000002 \\
0\end{array}$ \\
\hline 3.6 & 0.00011875 & $\begin{array}{c}0.0001187 \\
5\end{array}$ & 0.0000000 & $\begin{array}{c}0.0000773 \\
0\end{array}$ & $\begin{array}{c}0.0000773 \\
1\end{array}$ & $\begin{array}{c}0.0000000 \\
1\end{array}$ \\
\hline
\end{tabular}

Table 1. Comparison of $\mathrm{f}^{\prime}(\eta)$ from Devi and David (2012) result with the current research for $\mathrm{M}=0.4$ and $\mathrm{M}=0.8$

\begin{tabular}{|c|c|c|l|r|r|l|}
\hline$\theta(\eta)$ & \multicolumn{3}{|c|}{ M = 2.0 } & \multicolumn{3}{|c|}{ M = 4.0 } \\
\hline$\eta$ & $\begin{array}{l}\text { Devi \& } \\
\text { David }\end{array}$ & $\begin{array}{l}\text { Current } \\
\text { work }\end{array}$ & $\begin{array}{l}\text { Differen } \\
\text { ce }\end{array}$ & $\begin{array}{l}\text { Devi \& } \\
\text { David }\end{array}$ & $\begin{array}{l}\text { Current } \\
\text { work }\end{array}$ & $\begin{array}{l}\text { Differen } \\
\text { ce }\end{array}$ \\
\hline 0.0 & 1.000000 & 1.000000 & 0.000000 & 1.000000 & 1.000000 & 0.000000 \\
\hline 0.4 & 0.326922 & 0.326921 & 0.000001 & 0.277864 & 0.277864 & 0.000000 \\
\hline 0.8 & 0.106878 & 0.106877 & 0.000001 & 0.077208 & 0.077208 & 0.000000 \\
\hline 1.2 & 0.034941 & 0.034938 & 0.000003 & 0.021453 & 0.021453 & 0.000000 \\
\hline 1.6 & 0.011423 & 0.011420 & 0.000003 & 0.005961 & 0.005961 & 0.000000 \\
\hline 2.0 & 0.003734 & 0.003730 & 0.000004 & 0.001656 & 0.001656 & 0.000000 \\
\hline 2.4 & 0.001221 & 0.001215 & 0.000006 & 0.000460 & 0.000460 & 0.000000 \\
\hline 2.8 & 0.000399 & 0.000392 & 0.000007 & 0.000128 & 0.000127 & 0.000000 \\
\hline 3.2 & 0.000130 & 0.000122 & 0.000008 & 0.000036 & 0.000035 & 0.000001 \\
\hline 3.6 & 0.000043 & 0.000032 & 0.000011 & 0.000010 & 0.000008 & 0.000002 \\
\hline
\end{tabular}

Table 2. Comparison of $\theta(\eta)$ from Devi and David (2012) result with the current research for $\mathrm{M}=0.4$ and $\mathrm{M}=0.8$

There is good agreement in the comparison as presented in the tables. In contrast to the above numerical solution presented here, the Prandtl number used is one corresponding to the one for plasma ( $\mathrm{Pr}=0.71)$ and Schmidt number corresponding to that of water vapour $(\mathrm{Sc}=0.62)$.

\section{Results and Discussion}

The solutions to the dimensionless formulated equations governing the flow are computationally obtained for different physical parameters values entrenched in the model. The computational results are illustrated graphically for the various flow fields as showed in Fig 2 to Fig 15.

The impact of viscosity measuring term $\theta_{c}$ on the dimensionless momentum $f^{\prime}(\eta)$ and concentration field $\phi(\eta)$ are seen through Table 3 . As viscosity measuring parameter $\theta_{c}$ increases in magnitude, velocity decreases. Furthermore, it is interesting to notice that the rise in viscosity 
measuring term decrease the momentum boundary layer thickness. While rises in magnitude of viscosity measuring parameter $\theta_{\mathrm{c}}$ increases the concentration distribution. Thus, confirming the fact that the viscosity measuring parameter enhances the concentration boundary layer thickness. Table 4 shows the influence of viscosity measuring term $\theta_{c}$ over the temperature $\theta(\eta)$ and the effect of thermal conductivity measuring parameter $\theta_{r}$ on the dimensionless velocity field $f^{\prime}(\eta)$ respectively. Viscosity measuring parameter $\theta_{c}$ increases in magnitude, temperature increases. Also, as previously noted for concentration distribution, increase in viscosity measuring parameter enhances the temperature boundary layer thickness. While increases in magnitude of thermal conductivity measuring parameter $\theta_{\mathrm{r}}$ increases the velocity distribution. Also, it is noticed that the thermal conductivity measuring term enhances the flow rate boundary layer thickness. The influence of thermal conductivity measuring term on both concentration and heat distributions respectively is shown in Table 5. From this table, increase in thermal conductivity brings about decrease in concentration distribution. This is so because; increase in temperature will enhance consumption of more chemical species to support the rise in the momentum flow profile. Whereas, increase in thermal conductivity result to increase in temperature distribution. Thus, it is worth to mention that increase in thermal conductivity enhances the temperature and heat boundary layer.

\begin{tabular}{|c|c|c|c|c|c|c|c|c|}
\hline & \multicolumn{3}{|c|}{$\mathrm{f}^{\prime}(\eta)$} & \multicolumn{4}{c|}{$\phi(\eta)$} \\
\hline & $\theta_{\mathrm{c}}$ & & $\theta_{\mathrm{c}}$ & $\theta_{\mathrm{c}}$ & $\theta_{\mathrm{c}}$ & & $\theta_{\mathrm{c}}$ \\
$=1$ & $\theta_{\mathrm{c}}=5$ & $=100$ & $=200$ & $=0.1$ & $\theta_{\mathrm{c}}=5$ & $=100$ & $\begin{array}{c}\theta_{\mathrm{c}} \\
=200\end{array}$ \\
\hline & $=0.1$ & 1.0000 & 1.00000 & 1.00000 & 1.0000 & 1.0000 & 1.0000 & 1.0000 \\
0 & & & 0 & 0 & 0 & 0 \\
\hline 0. & 1.00000 & 0 & & & 0.7771 & 0.7949 & 0.7965 & 0.7966 \\
4 & 1.53506 & 6 & 1.27334 & 1.27210 & 7 & 4 & 6 & 5 \\
\hline 0. & & 1.2289 & & & 0.5558 & 0.5836 & 0.5862 & 0.5864 \\
8 & 1.25357 & 8 & 1.21920 & 1.21864 & 9 & 0 & 6 & 1 \\
\hline 1. & & 1.0306 & & & 0.3696 & 0.3975 & 0.4003 & 0.4005 \\
2 & 0.97260 & 3 & 1.03390 & 1.03410 & 4 & 5 & 5 & 1 \\
\hline 1. & & 0.8087 & & & 0.2314 & 0.2538 & 0.2561 & 0.2562 \\
6 & 0.72876 & 9 & 0.81759 & 0.81807 & 7 & 4 & 5 & 8 \\
\hline 2. & & 0.6058 & & & 0.1382 & 0.1537 & 0.1553 & 0.1554 \\
0 & 0.52800 & 6 & 0.61502 & 0.61553 & 1 & 0 & 2 & 1 \\
\hline
\end{tabular}

Table 3. Effect of viscosity measuring term on $\mathrm{f}^{\prime}(\eta)$ and $\phi(\eta)$

\begin{tabular}{|c|c|c|c|c|c|c|c|c|}
\hline & \multicolumn{9}{|c|}{$\theta(\eta)$} & \multicolumn{4}{c|}{$\mathrm{f}^{\prime}(\eta)$} \\
\hline & $\begin{array}{l}\theta_{\mathrm{c}} \\
=0.1\end{array}$ & $\theta_{\mathrm{c}}=5$ & $\begin{array}{l}\theta_{\mathrm{c}} \\
=100\end{array}$ & $\begin{array}{c}\theta_{\mathrm{c}} \\
=200\end{array}$ & $\begin{array}{c}\theta_{\mathrm{r}}=1 \\
\theta_{\mathrm{r}} \\
=20\end{array}$ & $\begin{array}{c}\theta_{\mathrm{r}} \\
=30\end{array}$ & $\begin{array}{c}\theta_{\mathrm{r}} \\
=40\end{array}$ \\
\hline & & 1.0000 & 1.00000 & 1.00000 & 1.0000 & 1.0000 & 1.0000 & 1.0000 \\
0 & & & 0 & 0 & 0 \\
\hline 0. & 1.00000 & 0 & & & 0.4049 & 1.4035 & 1.4170 & 1.4171 \\
4 & 0.88652 & 0 & 0.89044 & 0.89046 & 0 & 5 & 8 & 4 \\
\hline 0. & & 0.7725 & & & 1.2481 & 1.2465 & 1.2764 & 1.2765 \\
8 & 0.76552 & 1 & 0.77318 & 0.77322 & 0 & 3 & 2 & 4 \\
\hline 1. & & 0.6508 & & & 0.9857 & 0.9856 & 1.0335 & 1.0336 \\
2 & 0.64136 & 2 & 0.65175 & 0.65180 & 7 & 7 & 1 & 8 \\
\hline 1. & & 0.5301 & & & 0.7347 & 0.7375 & 0.8005 & 0.8007 \\
6 & 0.51951 & 1 & 0.53116 & 0.53122 & 6 & 5 & 3 & 2 \\
\hline & & 0.4161 & & & 0.5226 & 0.5290 & 0.6000 & 0.6002 \\
2 & 0.40577 & 3 & 0.41717 & 0.41723 & 0 & 6 & 6 & 5 \\
\hline
\end{tabular}


Table 4. Impact of viscosity measuring and thermal conductivity parameters on $\theta(\eta)$ and $f^{\prime}(\eta)$ respectively

\begin{tabular}{|c|c|c|c|c|c|c|c|c|}
\hline & \multicolumn{5}{|c|}{$\phi(\eta)$} & \multicolumn{4}{c|}{$\theta(\eta)$} \\
\hline$\eta$ & $\theta_{\mathrm{r}}=1$ & $\theta_{\mathrm{r}}=20$ & $\theta_{\mathrm{r}}=30$ & $\theta_{\mathrm{r}}=40$ & $\theta_{\mathrm{r}}=1$ & $\theta_{\mathrm{r}}=20$ & $\theta_{\mathrm{r}}=30$ & $\theta_{\mathrm{r}}=40$ \\
\hline 0 & 1.00000 & 1.00000 & 1.00000 & 1.00000 & 1.00000 & 1.00000 & 1.00000 & 1.00000 \\
\hline 0.4 & 0.79016 & 0.79007 & 0.78470 & 0.78468 & 0.88781 & 0.88641 & 0.89440 & 0.89445 \\
\hline 0.8 & 0.57653 & 0.57634 & 0.56659 & 0.56655 & 0.76572 & 0.76328 & 0.78220 & 0.78229 \\
\hline 1.2 & 0.39153 & 0.39124 & 0.37894 & 0.37890 & 0.63726 & 0.63443 & 0.66655 & 0.66669 \\
\hline 1.6 & 0.25060 & 0.25020 & 0.23744 & 0.23740 & 0.50783 & 0.50568 & 0.55154 & 0.55171 \\
\hline 2 & 0.15308 & 0.15261 & 0.14108 & 0.14104 & 0.38438 & 0.38425 & 0.44160 & 0.44178 \\
\hline
\end{tabular}

Table 5. Effect of thermal conductivity parameters on $\emptyset(\eta)$ and $\theta(\eta)$ respectively

We displayed the impact of magnetic interaction term (M) and radiation term (R) on the concentration distributions in Figures 2 and 3. Concentration distribution is encouraged with either rise in the radiation or magnetic field term. This implies that the species boundary layer thickens with a rise in $\mathrm{R}$ and $\mathrm{M}$. Increase in surface temperature and thermal buoyancy enhances the concentration field. The mass distribution is enhanced with variational rise in the surface temperature and thermal Grashof number as shown in Figure 4 and 5 respectively. While Figure 6 shows that mass distribution speedup with a rise in the generative chemical reaction $(\beta>0)$. The consequence of thermal radiation term ${ }^{\circledR}$ over the dimensionless heat $\theta(\eta)$ is seen through Fig.7. It is observed that the influence of radiation term is to decrease the heat distribution is the system. It elucidates that the thickness in the boundary layer of the energy equation is reducing as $R$ values enhance. The response of heat source term in the heat equation to a rise in the magnetic field $M$ over the flow heat $\theta(\eta)$ is depicted with the aid of Fig. 8. Enhancing the magnetic field term $\mathrm{M}$ encourages Lorentz force in the flow which then resulted into rise in the heat profile. This described the fact that increase in the Lorentz force boosted the temperature source term in the energy balance equation. The effect of stretching surface temperature term $\theta_{w}$ on the dimensionless heat $\theta(\eta)$ is presented as Figure 9. Rising in the surface heat term $\theta_{w}$ is seen to have increasing effect on the temperature distribution. Figure 10 illustrates thermal buoyancy effect on the temperature field. Boosting the thermal Grashof number (Grt) decreases the temperature distribution, this describing the impact of Grt on the bulk temperature. Decreasing in the bulk temperature influence the heat source parameter in the system to increases thereby enhances the heat profile. Figures 12 and 13 portray the graphical result of the momentum $\mathrm{f}^{\prime}(\eta)$ for various values of radiation term $R$ and magnetic interaction parameter $M$. It is observed that as the parameter values $R$ and $M$ rises, the fluid velocity distribution $f^{\prime}(\eta)$ reduces to show influence of radiation and magnetic field terms on the fluid flow rate in under different variable properties. The profiles decelerated as the parameters values in boosting. The action of surface temperature and thermal buoyancy on non-dimensional velocity distribution in plotted in Figures 14 and 15 respectively. It is obtained from the plots that an increase in either $\theta_{\mathrm{w}}$ or Grt increases the flow momentum and enhances thickness of the flow boundary layer as seen due to a rise in the values of $\theta_{\mathrm{w}}$ or Grt. 


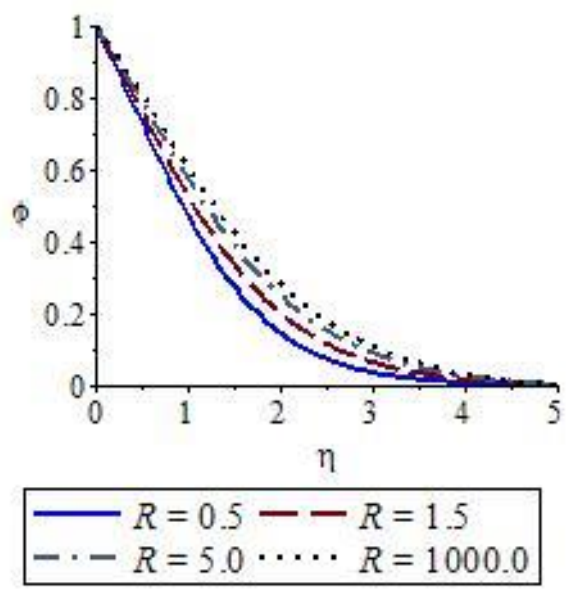

Fig. 2. Concentration distributions profile for various radiation parameters $R$

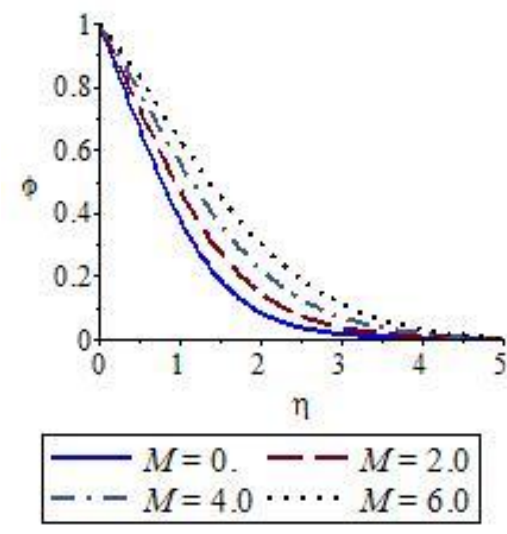

Fig. 3. Concentration distributions profile for various $M$

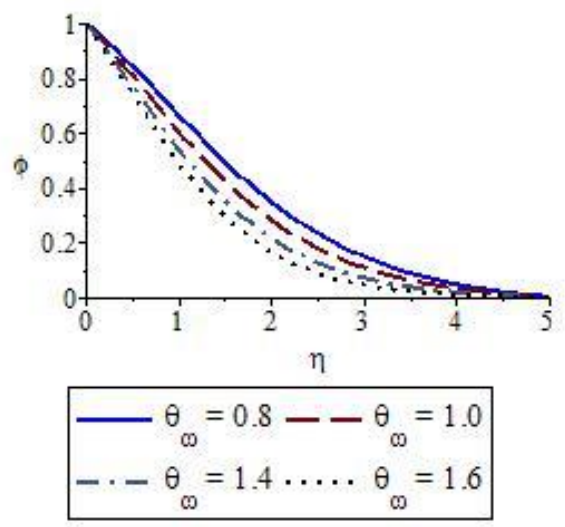

Fig. 4. Concentration distributions profile for various $\theta_{\mathrm{w}}$ 


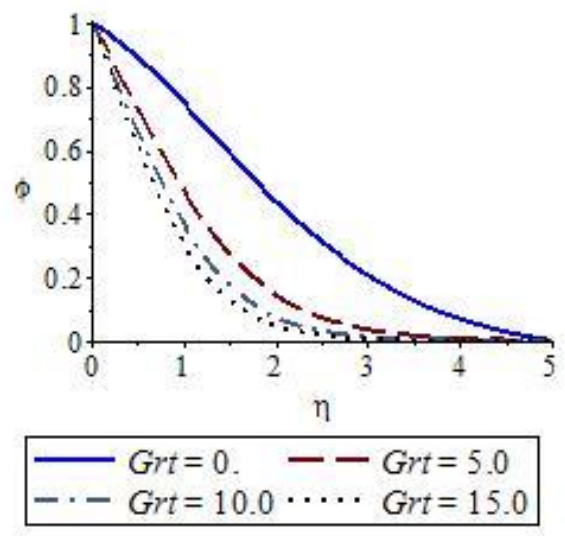

Fig. 5. Concentration distributions profile for various Grt

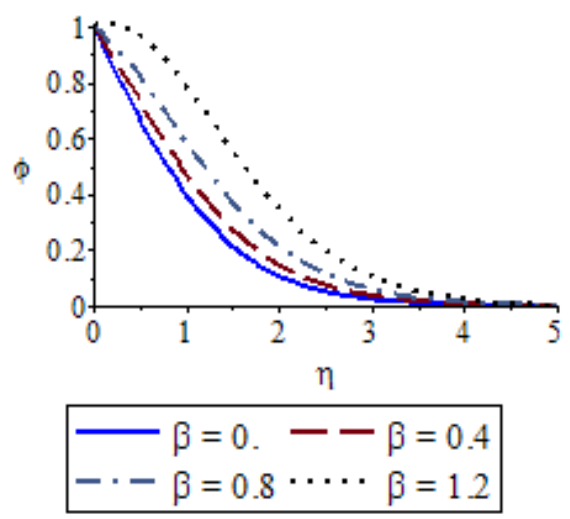

Fig. 6. Concentration distributions profile for various $\beta$

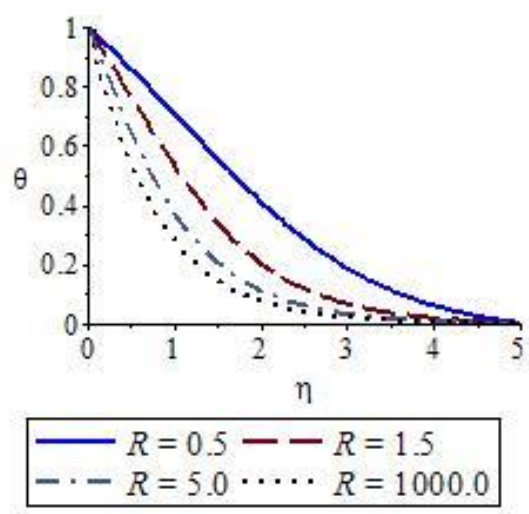

Fig. 7. Temperature distributions profile for various $\mathrm{R}$ 


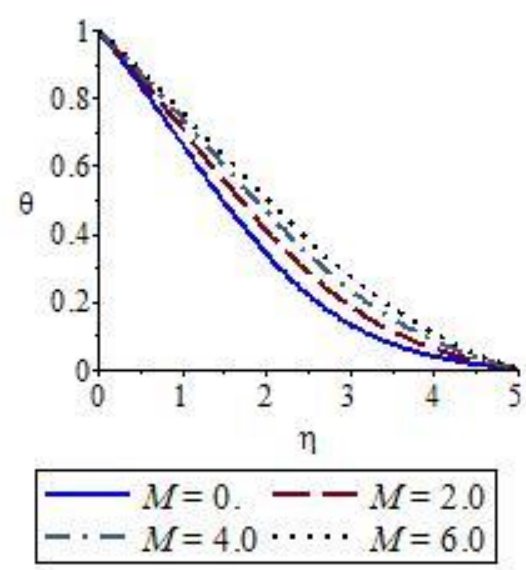

Fig. 8. Temperature distributions profile for various $\theta_{w}$

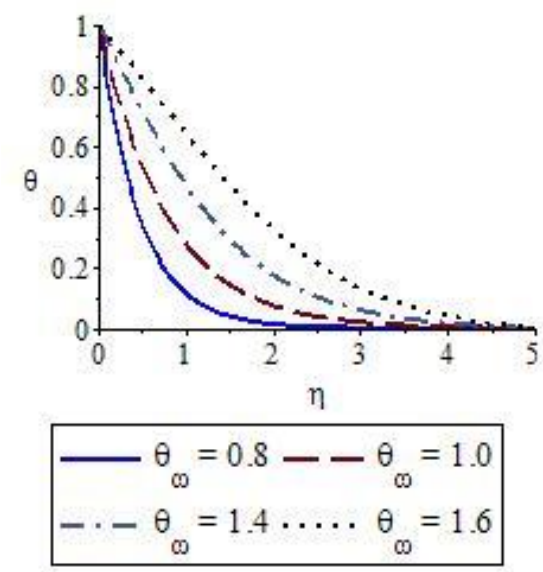

Fig. 9. Temperature distributions profile for various $M$

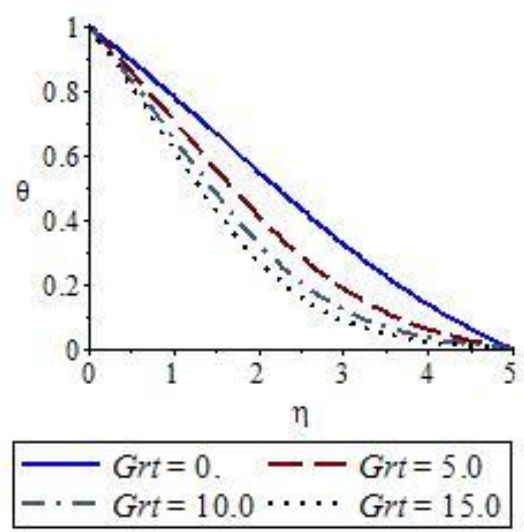

Fig. 10. Temperature distributions profile for various Grt 


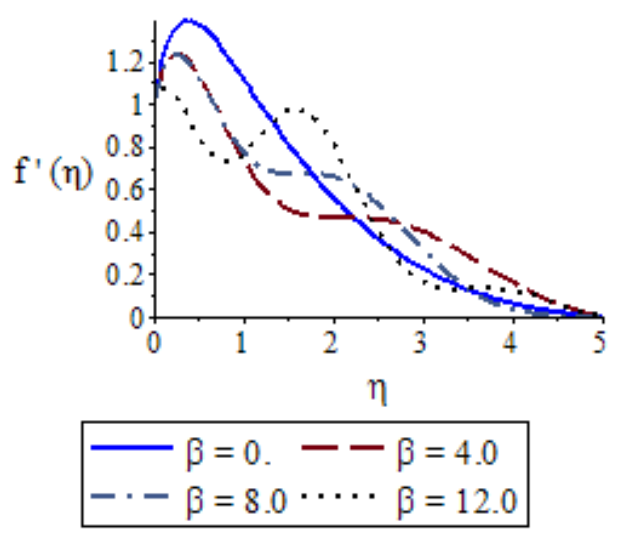

Fig. 11. Velocity distributions profile for various $\beta$

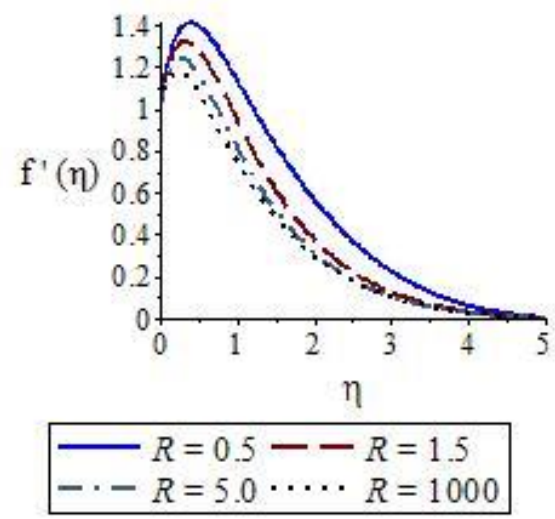

Fig. 12. Velocity distributions profile for various $R$

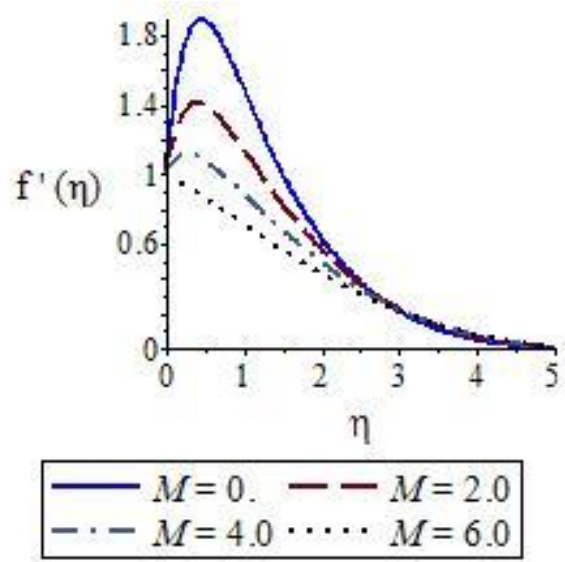

Fig. 13. Velocity distributions profile for various $M$ 


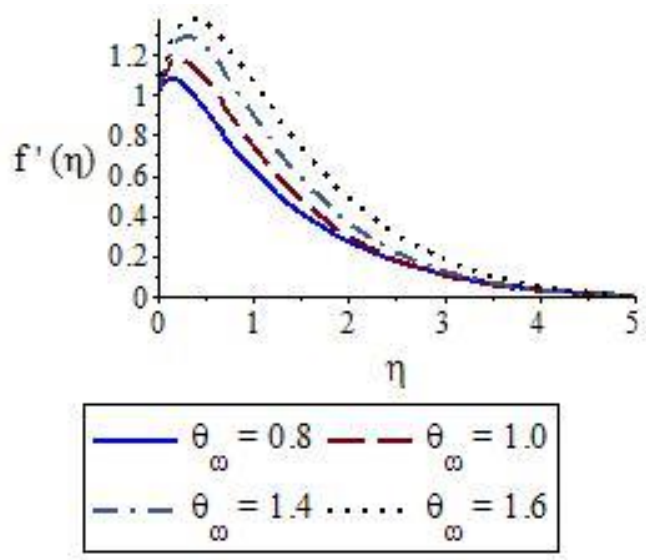

Fig. 14. Velocity distributions profile for various $\theta_{\mathrm{w}}$

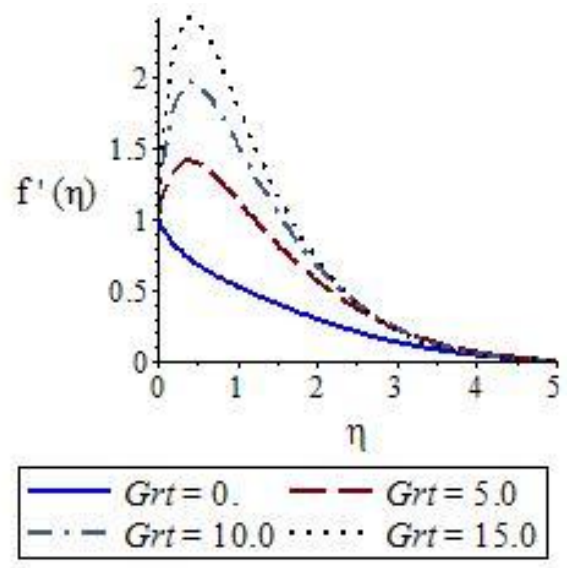

Fig. 15. Velocity distributions profilefor various Grt

\section{Skin friction, heat and mass gradient}

We now move to examining some important fluid parameters that are of importance to this work. Such parameters include Skin friction, Sherwood and Nusselt numbers coefficient. We therefore denote and define respectively, Skin friction, Nusselt and Sherwood numbers as Salawu and Dada (2016);

$$
c_{f}=\frac{T_{f}}{\rho u_{\omega} v_{\omega}}, T_{f}=\left.\mu \frac{d u}{d y}\right|_{y=0} \Rightarrow c_{f}=-\frac{d^{2}}{d \eta^{2}} f(0)
$$

The heat transfer at the wall is computed from Fourier's law:

$$
N u=\frac{q_{\omega} v}{\left(T_{\omega}-T_{\infty}\right) K v_{\omega}}, q_{\omega}=-\left.K \frac{d T}{d y}\right|_{y=0} \Rightarrow S h=-\frac{d}{d \eta} \phi(0)
$$

And mass transfer rate at wall 


$$
S h=\frac{J_{\omega} v}{\left(J_{\omega}-J_{\infty}\right) D v_{\omega}}, J_{\omega}=-\left.D \frac{d C}{d y}\right|_{y=0} \Rightarrow S h=-\frac{d}{d \eta} \phi(0)
$$

Table 6 presents the effect of the parameters Grt, Grc, M, $\theta_{c}, \theta_{r}, \theta_{w}, R, \beta$ on the wall shear stress $\mathrm{c}_{\mathrm{f}}$, Sherwood number $\mathrm{Sh}$ and wall heat gradient $\mathrm{Nu}$ for $\mathrm{Sc}=0.62, \operatorname{Pr}=0.71$ and $\epsilon=0.01$. From the table, the heat and spaces buoyancy, surface temperature and reaction parameter rises the coefficient of skin friction $\mathrm{c}_{\mathrm{f}}$. But thermal and viscosity conductivity measuring heat transport and parameters reduces the coefficient skin friction $\mathrm{c}_{\mathrm{f}}$. It is also noticed that the dimensionless wall heat transport gradient $\mathrm{Nu}$ enhances with rises in the thermal and mass buoyancy, thermal conductivity measuring parameter and heat transfer rate, but decrease with viscosity measuring parameter, surface temperature, magnetic and chemical reaction parameters. While surface temperature, thermal and mass buoyance are found to enhance the mass transfer rate at the wall and decreases with Hartmann number, thermal and viscosity conductivity measuring parameter, heat transfer rate and chemical reactivity terms.

\begin{tabular}{|c|c|c|c|c|c|c|c|c|c|c|}
\hline Grt & Grc & $\mathrm{M}$ & $\theta_{c}$ & $\theta_{\mathrm{r}}$ & $\theta_{\mathrm{w}}$ & $\mathrm{R}$ & $\mathrm{B}$ & $c_{f}$ & $\mathrm{Sh}$ & $\mathrm{Nu}$ \\
\hline 0.0 & 2.0 & 2.0 & 0.6 & 0.8 & 1.5 & 5.0 & 0.4 & -1.08182 & 0.15662 & 0.20182 \\
\hline 5.0 & 2.0 & 2.0 & 0.6 & 0.8 & 1.5 & 5.0 & 0.4 & 2.57279 & 0.49550 & 0.26680 \\
\hline 10.0 & 2.0 & 2.0 & 0.6 & 0.8 & 1.5 & 5.0 & 0.4 & 5.86878 & 0.64549 & 0.31049 \\
\hline 15.0 & 2.0 & 2.0 & 0.6 & 0.8 & 1.5 & 5.0 & 0.4 & 8.92490 & 0.74383 & 0.34283 \\
\hline 5.0 & 0.0 & 2.0 & 0.6 & 0.8 & 1.5 & 5.0 & 0.4 & 1.25845 & 0.43265 & 0.25230 \\
\hline 5.0 & 2.0 & 2.0 & 0.6 & 0.8 & 1.5 & 5.0 & 0.4 & 2.57279 & 0.49550 & 0.26680 \\
\hline 5.0 & 6.0 & 2.0 & 0.6 & 0.8 & 1.5 & 5.0 & 0.4 & 4.99743 & 0.58459 & 0.28893 \\
\hline 5.0 & 12.0 & 2.0 & 0.6 & 0.8 & 1.5 & 5.0 & 0.4 & 8.32460 & 0.67732 & 0.31370 \\
\hline 5.0 & 2.0 & 0.0 & 0.6 & 0.8 & 1.5 & 5.0 & 0.4 & 4.83244 & 0.62428 & 0.30337 \\
\hline 5.0 & 2.0 & 2.0 & 0.6 & 0.8 & 1.5 & 5.0 & 0.4 & 2.57279 & 0.49550 & 0.26680 \\
\hline 5.0 & 2.0 & 4.0 & 0.6 & 0.8 & 1.5 & 5.0 & 0.4 & 0.95431 & 0.38386 & 0.24054 \\
\hline 5.0 & 2.0 & 6.0 & 0.6 & 0.8 & 1.5 & 5.0 & 0.4 & -0.27328 & 0.28910 & 0.22220 \\
\hline 5.0 & 2.0 & 2.0 & 0.1 & 0.8 & 1.5 & 5.0 & 0.4 & 5.95466 & 0.52346 & 0.27171 \\
\hline 5.0 & 2.0 & 2.0 & 50.0 & 0.8 & 1.5 & 5.0 & 0.4 & 1.34764 & 0.47182 & 0.26257 \\
\hline 5.0 & 2.0 & 2.0 & 100 & 0.8 & 1.5 & 5.0 & 0.4 & 1.33821 & 0.47158 & 0.26252 \\
\hline 5.0 & 2.0 & 2.0 & 2000 & 0.8 & 1.5 & 5.0 & 0.4 & 1.32921 & 0.47135 & 0.26248 \\
\hline 5.0 & 2.0 & 2.0 & 0.6 & 0.0 & 1.5 & 5.0 & 0.4 & 2.56594 & 0.48865 & 0.26652 \\
\hline 5.0 & 2.0 & 2.0 & 0.6 & 0.1 & 1.5 & 5.0 & 0.4 & 2.56234 & 0.48889 & 0.27025 \\
\hline 5.0 & 2.0 & 2.0 & 0.6 & 15 & 1.5 & 5.0 & 0.4 & 2.59287 & 0.50270 & 0.25392 \\
\hline 5.0 & 2.0 & 2.0 & 0.6 & 40 & 1.5 & 5.0 & 0.4 & 2.59302 & 0.50275 & 0.25381 \\
\hline 5.0 & 2.0 & 2.0 & 0.6 & 0.8 & 0.8 & 5.0 & 0.4 & 1.24735 & 0.25659 & 1.79983 \\
\hline 5.0 & 2.0 & 2.0 & 0.6 & 0.8 & 1.0 & 5.0 & 0.4 & 1.78345 & 0.33036 & 1.12815 \\
\hline 5.0 & 2.0 & 2.0 & 0.6 & 0.8 & 1.2 & 5.0 & 0.4 & 2.22678 & 0.40798 & 0.56860 \\
\hline 5.0 & 2.0 & 2.0 & 0.6 & 0.8 & 1.4 & 5.0 & 0.4 & 2.48936 & 0.47156 & 0.33215 \\
\hline 5.0 & 2.0 & 2.0 & 0.6 & 0.8 & 1.5 & 0.5 & 0.4 & 2.57279 & 0.49550 & 0.26680 \\
\hline 5.0 & 2.0 & 2.0 & 0.6 & 0.8 & 1.5 & 1.5 & 0.4 & 2.33973 & 0.42677 & 0.44214 \\
\hline 5.0 & 2.0 & 2.0 & 0.6 & 0.8 & 1.5 & 5.0 & 0.4 & 2.06203 & 0.36876 & 0.70642 \\
\hline 5.0 & 2.0 & 2.0 & 0.6 & 0.8 & 1.5 & 2000 & 0.4 & 1.78543 & 0.33059 & 1.12413 \\
\hline 5.0 & 2.0 & 2.0 & 0.6 & 0.8 & 1.5 & 5.0 & 0.0 & 2.51671 & 0.68072 & 0.26486 \\
\hline 5.0 & 2.0 & 2.0 & 0.6 & 0.8 & 1.5 & 5.0 & 0.4 & 2.57279 & 0.49550 & 0.26680 \\
\hline 5.0 & 2.0 & 2.0 & 0.6 & 0.8 & 1.5 & 5.0 & 0.8 & 2.65253 & 0.25056 & 0.26982 \\
\hline 5.0 & 2.0 & 2.0 & 0.6 & 0.8 & 1.5 & 5.0 & 1.2 & 2.78307 & 0.12245 & 0.27518 \\
\hline
\end{tabular}

Table 6. Skin friction, gradient of energy and species transfer 


\section{Conclusion}

The following conclusions are made in view of the above obtained results.

- It is noticed that the magnetic field decelerates the momentum and enhances temperature distribution in the system under consideration.

- It is seen that the term that increases the heat source term diminishes the fluid viscosity.

- Temperature is found to reduce due to the radiation term effect while it is found to increase with the increasing surface heat parameter.

- The thickness of heat boundary layer reduces significantly as Prandtl number rises.

- The skin friction and the fluid flow rate decrease by the velocity exponent term. On the other hand, heat diffusion is enhanced by the velocity exponent parameter.

- It is obtained that for encouraging radiation parameter $\mathrm{R}$, the dimensionless rate of heat transfer decreases.

\section{References}

Abdou MMM (2010). Effect of radiation with temperature dependent viscosity and thermal conductivity on unsteady a stretching sheet through porous media, Nonlinear Analysis: Modeling and Control, 15(3). 257-270.

Chen Ch H (2006). Effect of viscous dissipation on heat transfer in a non-Newtonian liquid film over an unsteady stretching sheet, J. Non-Newton fluid, 135(2-3), 128-135.

Devi SP, Gururaj DM (2012). Effects of variable viscosity and nonlinear radiation on MHD flow with heat transfer over a surface stretching with a power-law velocity. Advances in Applied Science Research, 3 (1), 319-334.

Hassan AR, Gbadeyan JA, Salawu SO (2018). The effects of thermal radiation on a reactive hydromagnetic internal heat generating fluid flow through parallel porous plates. Springer Proceedings in Mathematics \& Statistics, 259, 183-193.

Hayat T, Shehzad SA, Alsaedi A. (2013). Three-dimensional stretched flow of Jeffrey fluid with variable thermal conductivity and thermal radiation. Appl. Math. Mech. Engl. Ed., 34(7), 823-832.

Hazarika G C, Gopal US (2012). Effects of variable viscosity and thermal conductivity on MHD flow past a vertical plate. Escuela Regional de Matematicas, 20(2), 45-54.

Kareem RA, Salawu SO, Yubin Yan (2020). Analysis of transient Rivlin-Ericksen fluid and irreversibility

of exothermic reactive hydromagnetic Variable Viscosity. J. Appl. Comput. Mech., 6(1), 2636.

Kareem RA, Salawu SO, Gbadeyan JA (2018). Numerical analysis of non-uniform heat Source/Sink in a radiative micropolar variable electric conductivity fluid with dissipation Joule heating. American Journal of Applied Mathematics, 6(2), 34-41.

Modather M, Abdou M, EL-Kabeir, SMM (2007). Magnetohydrodynamics and radiative effect on free convection flow of fluid with variable viscosity from a vertical plate through a porous medium, J. Porous Media, 10(5), 503-556.

Okedoye AM (2015). On the unsteady free convective flow with Radiative heat transfer of Sisko fluid. Int. J. of Innovative Science, Engineering \& Technology, 2(5), 427-437.

Okedoye AM, Akinrinmade VA, Onifade YS (2017). Heat and mass transfer MHD flow of a Sisko fluid in porous medium. Confluence Journal of Pure and Applied Science, 11(1), 324333. 
Pantokratoras A (2006). The effect of variable viscosity on mixed convection heat transfer along a vertical moving surface, Int. J. of Thermal Sciences, 45, 60-69, (2006).

Patowary G (2012). Effect of variable viscosity and thermal conductivity of micropolar fluid in a porous channel in presence of magnetic field. International Journal for Basic Sciences and Social Sciences, 1(3), 69-77.

Rani HP, Reddy GJ, Kim CN (2013). Transient analysis of diffusive chemical reactive species for couple stress fluid flow over vertical cylinder. Appl. Math. Mech. Engl. Ed., 34(8), 9851000 .

Salawu SO, Ogunseye HA (2020). Entropy generation of a radiative hydromagnetic PowellEyring chemical reaction nanofluid with variable conductivity and electric field loading. Results in Engineering, 5, 100072.

Salawu SO (2018). Analysis of third-grade heat absorption hydromagnetic exothermic chemical reactive flow in a Darcy-forchheimer porous medium with convective cooling. WSEAS Transaction on Mathematics, 17, 280-289.

Salawu SO, Dada MS (2018). Lie group analysis of soret and dufour effects on radiative inclined magnetic pressure-driven flow past a Darcy-forchheimer medium. Journal of the Serbian Society for Computational Mechanics, 12(1), 108-125.

Salawu SO, Fatunmbi EO (2017). Inherent Irreversibility of Hydromagnetic Third-Grade Reactive Poiseuille Flow of a Variable Viscosity in Porous Media with Convective Cooling. Journal of the Serbian Society for Computational Mech. 11, 46-58.

Salawu SO, Ogunseye HA, Olanrewaju AM (2018). Dynamical analysis of unsteady Poiseuille flow of two-step exothermic non-Newtonian chemical reactive fluid with variable viscosity. Int. J. of Mech. Eng. and Tech., 9(12), 596-605.

Salawu SO, Okedoye AM (2018). Thermodynamic second law analysis of hydromagnetic gravity-driven two-step exothermic chemical reactive flow with heat absorption along a channel. Iranian Journal of Energy and Environment, 9(2), 114-120.

Salawu SO, Dada MS (2016). Radiative heat transfer of variable viscosity and thermal conductivity effects on inclined magnetic field with dissipation in a non-Darcy medium. Journal of the Nigerian Mathematical Society 35, 93-106.

Salawu SO, Oke SI (2018). Inherent irreversibility of exothermic chemical reactive third-grade Poiseuille flow of a variable viscosity with convective cooling. J. Appl. Comput. Mech., 4(3), 167-174.

Salem AM (2007). Variable viscosity and thermal conductivity effects on MHD flow and heat transfer in viscoelastic fluid over a stretching sheet, Phys. Letter, A, 369 (4), 315-322.

Seth GS, Hussain SM, Singh JK (2011). MHD Couette flow of class-II in a rotating system. J. Appl. Math. and Bioinformatics, 1, 31-54.

Sharidan S, Mahmood T, Pop I. (2016) Similarity solution for the unsteady boundary layer flow and heat transfer due to a stretching sheet, Int. J. Appl. Mech. Mech. Eng., 11(3), 647-654, (2006).

Yurusoy M (2006). Unsteady boundary layer flow of power-law fluid on stretching sheet surface, Int. J. Eng. Sci., 44 (5- 6), 325-332. 